\title{
Neutrophil-to-lymphocyte and platelet-to- lymphocyte ratios as predictive and prognostic markers in patients with locally advanced rectal cancer treated with neoadjuvant chemoradiation
}

\author{
Shaan Dudani ${ }^{1,5^{*}} \mathbb{D}$, Horia Marginean ${ }^{1}$, Patricia A. Tang ${ }^{2}$, Jose G. Monzon², Soundouss Raissouni ${ }^{3}$,
} Timothy R. Asmis ${ }^{1}$, Rachel A. Goodwin ${ }^{1}$, Joanna Gotfrit ${ }^{1}$, Winson Y. Cheung ${ }^{4,5}$ and Michael M. Vickers ${ }^{1}$

\begin{abstract}
Background: A standard therapy for locally advanced rectal cancer (LARC) includes fluoropyrimidine (FP)-based neoadjuvant chemoradiation (nCRT). Previous studies have inconsistently demonstrated that baseline neutrophil- and platelet-to-lymphocyte ratios (NLR and PLR) are predictive of response to nCRT or prognostic of outcomes in LARC.

Methods: We reviewed patients with LARC undergoing nCRT followed by surgery from 2005 to 2013 across 8 Canadian cancer centres. Outcome measures of interest were pathological complete response (pCR), disease-free survival (DFS) and overall survival (OS). Logistic regression and Cox proportional hazard models were used to assess for associations between baseline hematologic variables and outcomes.

Results: Of 1527 identified patients, 1237 (81\%) were included in the DFS/OS analysis. Median age was 62 (range 23-88), $69 \%$ were male, and $80 \%$ had performance status (PS) $0-1$. Twenty-six percent had elevated NLR ( $\geq 4$ ), and 66\% had elevated PLR ( $\geq 150$ ). Ninety-seven percent of patients received FP-based nCRT, with $96 \%$ receiving $\geq 44$ Gy. $81 \%$ completed neoadjuvant chemotherapy and 95\% completed neoadjuvant radiotherapy, with a pCR rate of $18 \%$. After a median follow-up time of 71 months, $8 \%$ developed local recurrence, 22\% developed distant recurrence and 24\% died. 5-year DFS and OS were 69\% (95\% Cl 66-72\%) and 79\% (95\% Cl 77-82\%), respectively. In multivariate analyses, elevated baseline NLR and PLR were neither prognostic for DFS and OS nor predictive of $\mathrm{PCR}$.
\end{abstract}

Conclusions: NLR and PLR were not found to be independently prognostic for DFS or OS and did not predict for pCR in patients with LARC undergoing nCRT followed by surgery.

Keywords: Biomarkers, Survival, Pathologic complete response, Inflammatory response, Personalized medicine

\section{Background}

A standard of care treatment for locally advanced rectal cancer (LARC) includes fluoropyrimidine-based concurrent neoadjuvant chemoradiotherapy (nCRT) followed by total mesorectal excision (TME) $[1,2]$. As compared to post-

\footnotetext{
* Correspondence: shaan.dudani@gmail.com

${ }^{1}$ The Ottawa Hospital Cancer Centre/University of Ottawa, Ottawa, Ontario, Canada

${ }^{5}$ Present address: Division of Medical Oncology, Tom Baker Cancer Centre, University of Calgary, 133129 St NW, Calgary, AB T2N 4N2, Canada Full list of author information is available at the end of the article
}

operative chemoradiation, use of nCRT in LARC is associated with improved rates of local control, tumour downstaging and sphincter-sparing surgery, as well as an improved toxicity profile [3]. However, response to nCRT varies widely between patients. Although roughly three-quarters of patients demonstrate evidence of response on postoperative histopathologic evaluation, with a proportion (usually $<25 \%$ ) demonstrating pathologic complete response (pCR), up to one quarter of patients exhibit resistance to nCRT, displaying either minimal regression or complete lack of response [4-6]. Patients with radiographic and/or pathologic

(c) The Author(s). 2019 Open Access This article is distributed under the terms of the Creative Commons Attribution 4.0 International License (http://creativecommons.org/licenses/by/4.0/), which permits unrestricted use, distribution, and 
evidence of response to neoadjuvant therapy have been demonstrated to have improved long-term outcomes, including disease-free survival (DFS) and overall survival (OS) [5-9].

The mechanism(s) underlying the observed heterogeneity of tumour sensitivity to nCRT are not well understood, and currently there are no effective pre-operative models or biomarkers to predict response to nCRT. The ability to predict response and prognosis in patients undergoing nCRT for LARC could allow for cancer-directed treatments to be delivered in a more individualized manner. For example, patients predicted to have exquisite sensitivity to nCRT may be candidates for emerging "watch and wait", organpreserving strategies that may spare patients from the significant morbidity associated with rectal surgery [10-13]. Alternatively, patients with tumours predicted to be resistant to nCRT may be candidates for alternate neoadjuvant approaches (such as 'total neoadjuvant therapy', which incorporates preoperative chemotherapy in addition to nCRT [14]) or be considered for treatment with upfront surgery.

A range of clinical, radiologic, serologic, histopathologic and genetic factors have been studied as potential predictors of response to nCRT in LARC $[8,15-18]$. Among these, the neutrophil-to-lymphocyte ratio (NLR) and platelet-to-lymphocyte ratio (PLR) are two readilyavailable serologic biomarkers which are felt to be surrogates for the degree of systemic inflammation and have been studied as prognostic markers in a range of malignancies $[19,20]$. Previous studies have yielded conflicting results as to their prognostic/predictive potential in rectal cancer [19-28], though few studies have compared the roles of NLR and PLR in the same cohort. We conducted a multi-institutional review to assess the ability of NLR and PLR to predict prognosis and likelihood of response in patients with LARC treated with nCRT.

\section{Methods}

\section{Study design and patient selection}

Patients were identified and data were extracted from the Canadian Health Outcomes Research Database (CHORD) Consortium's Rectal Cancer Database, which is a national, multi-institutional registry of locally advanced rectal cancer patients who have undergone nCRT followed by curative intent-surgery from four academic (British Columbia Cancer Agency, Cross Cancer Institute, The Ottawa Hospital Cancer Centre, Tom Baker Cancer Centre) and four community (Central Alberta Cancer Centre, Grand Prairie Cancer Centre, Jack Ady Cancer Centre, Margery E. Yuill Cancer Centre) cancer centres in Canada.

Patients were eligible for inclusion if they had: pathologically-confirmed rectal adenocarcinoma; clinical stage II or III disease as per the seventh edition of the American Joint Commission on Cancer staging system [29]; commenced long-course nCRT; underwent curative- intent surgery; baseline hematologic markers available (within 4 weeks prior and 2 weeks after initiating nCRT); documented absence of metastases (confirmed by CT or MRI of the abdomen and either chest radiograph or CT thorax). Patients were excluded if they had prior treatments for rectal cancer, evidence of metastatic disease, did not receive surgery, or received neoadjuvant radiation alone.

\section{Baseline hematologic variables}

NLR was calculated by dividing the absolute neutrophil count by the absolute lymphocyte count. PLR was calculated by dividing the platelet count by the absolute lymphocyte count. NLR was defined as elevated if $\geq 4$ and PLR was defined as elevated if $\geq 150$. These cutpoints were chosen based on systematic reviews of prior studies that used these thresholds and established them to be potentially predictive/prognostic $[19,20]$. Restricted cubic spline analysis was also used to assess non-linear associations between NLR/PLR levels and survival endpoints, to determine if NLR $\geq 4$ and $P L R \geq$ 150 were appropriate cut-points [30].

\section{Statistical analysis}

We summarized patients' demographics and baseline characteristics using descriptive statistics. When missing data were encountered, continuous variables were categorized and missing data were coded as not available (NA). Outcome measures of interest included DFS, OS and pCR. DFS was defined as time from diagnosis to first event (local recurrence, distant recurrence, or death from any cause) and censored at the date of last followup. OS was defined as the time from diagnosis to death from any cause and censored at the date of last followup. pCR was defined as the absence of any residual tumour cells on post-operative histologic evaluation of the rectal surgical specimen.

DFS and OS were evaluated using the Kaplan-Meier method. Uni- and multi-variable Cox regressions were conducted to determine the prognostic value of NLR and PLR on outcomes (DFS, OS) after adjustment for confounders. The assumptions of proportional hazards were checked for all final models. The covariate 'province' did not meet the assumption of the Cox regression. 'Adjuvant chemotherapy' was associated with lower risk of death but also did not meet the assumption of proportional hazards required for valid inference when using Cox proportional hazards. As a result, multivariable survival analysis was performed by stratifying on the two variables 'province' and 'adjuvant chemotherapy'. A logistic regression model was also constructed to explore NLR and PLR as independent predictors of pCR.

The covariates were screened using univariate analyses and dropped from further inclusion in multivariate models if their crude association's $p$-value with the outcomes was 
$>0.2$. The remaining variables and their interactions were combined in a multivariate model. The interaction terms were assessed first for elimination from the model using a likelihood ratio test, significant at the $10 \%$ level $(p=0.1)$. The covariates were then assessed utilizing 2 methods: i) significance at the $10 \%$ level $(\mathrm{p}=0.1)$ and, ii) the $10 \%$ change-in-estimate approach, where a variable was kept in the multivariate model if significant and its exclusion resulted in a substantial (>10\%) change to the survival coefficient estimate. Factors significant at the 0.05 level were retained in the multivariate model. Akaike's Information Criterion and Bayesian Information Criterion were used to select the best models for NLR and PLR effect on outcomes.

Estimates (hazard ratios, odds ratios) are presented with 95\% confidence intervals (95\% CIs). We considered a $p$-value of 0.05 to be significant. All statistical analyses were performed using Stata $^{\oplus}$ software, version 13.1 (Stata Corp LP, College Station, TX).

\section{Results}

\section{Patient and tumour characteristics}

Of 1527 identified patients, 1237 (81\%) met eligibility criteria and were included for analysis (Fig. 1). All ineligible patients were excluded due to having unavailable baseline hematologic data. Patient demographics and tumour characteristics are summarized in Table 1.

The median age was 62 (range 23-88), with 69\% male and $80 \%$ Eastern Cooperative Oncology Group (ECOG) performance status $0-1$. Twenty-six percent had NLR $\geq 4$ and $66 \%$ had PLR $\geq 150$. Median pre-treatment carcinoembryonic antigen (CEA) level was $4 \mathrm{ng} / \mathrm{ml}$. Clinical stage II and III disease was noted in 28 and $71 \%$ of patients, respectively. Patients were followed for a median of 71 months.

\section{Treatments}

Median radiation dose received was 50 Gy (range 2080 ), with $96 \%$ receiving $\geq 44$ Gy. Ninety-seven percent of patients received fluoropyrimidine-based neoadjuvant chemotherapy (35\% capecitabine, 62\% 5-fluorouracil), while $1 \%$ received neoadjuvant raltitrexed ( $2 \%$ not reported). Neoadjuvant chemotherapy and radiotherapy were completed as planned in 81 and 95\% of patients, respectively. Fifty-three percent of patients underwent low anterior resection, $43 \%$ underwent abdominoperineal resection, and $3 \%$ underwent pelvic exenteration. Circumferential resection margin was $>1 \mathrm{~mm}$ (uninvolved) in $86 \%, \leqslant 1 \mathrm{~mm}$ (involved) in $8 \%$, and unknown in $6 \%$. The majority (80\%) underwent TME within 6-12 weeks of completion of nCRT. Adjuvant chemotherapy was used in $73 \%$ of patients, with $28 \%$ of the total group receiving oxaliplatin-based adjuvant chemotherapy. Treatment details are summarized in Table 2.

\section{Outcomes}

After a median follow-up time of 71 months, $8 \%$ developed local recurrence (LR), 22\% developed distant recurrence (DR) and 24\% had died. Median DFS was 132 months (95\% CI 127 months - not reached), while median OS was not reached. 5-year DFS and OS rates were 69\% (95\% CI 66-72\%) and 79\% (95\% CI 77-82\%), respectively. pCR rate was $18 \%$.

\section{Univariate and multivariate analyses}

Factors included in univariate analyses were: age, sex, province, year of diagnosis (pre vs. post 2010), body mass

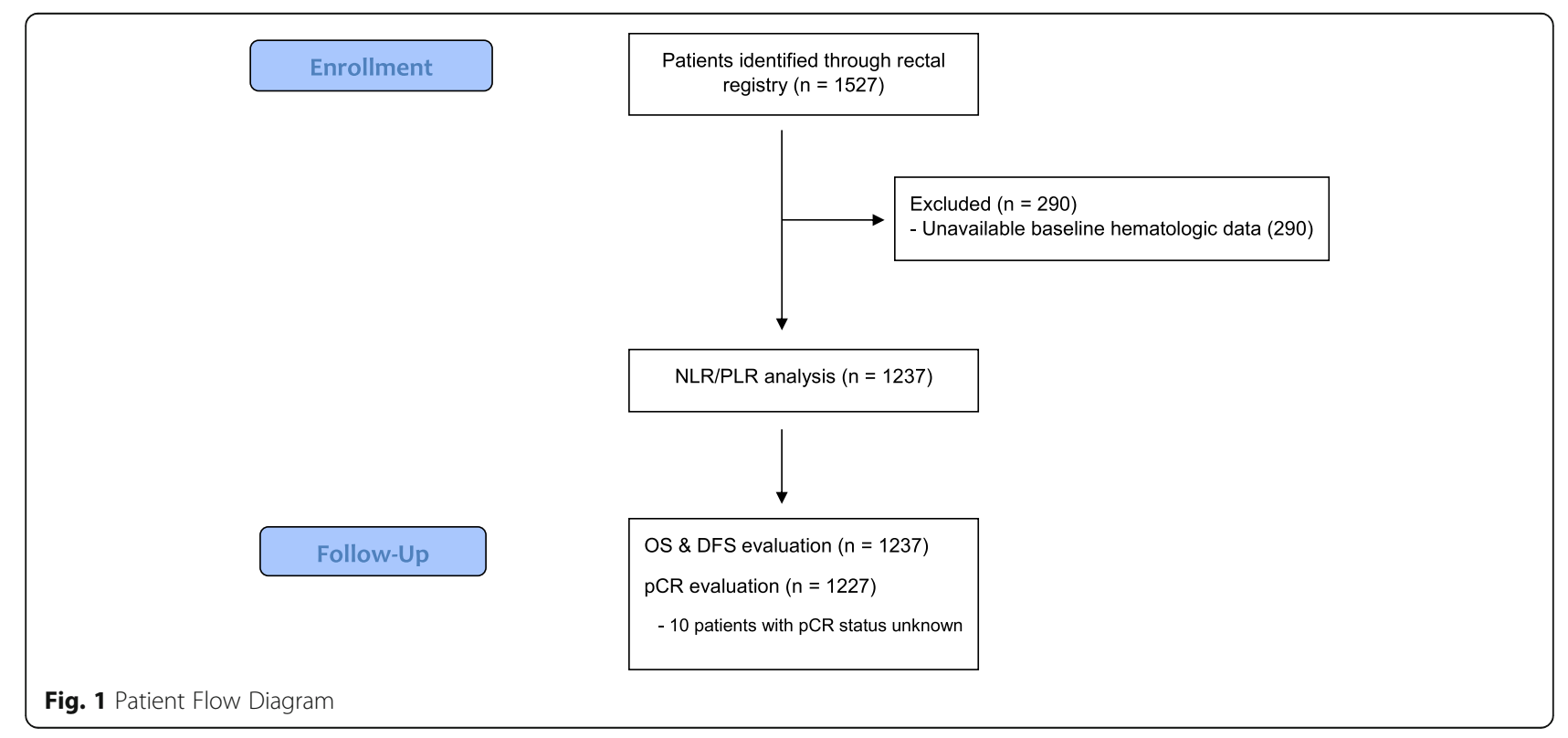


Table 1 Baseline Patient and Tumour Characteristics

\begin{tabular}{|c|c|c|c|c|c|c|c|c|}
\hline \multirow[t]{2}{*}{ Variable } & & \multirow{2}{*}{$\begin{array}{l}\text { Total }(n= \\
1237)\end{array}$} & \multicolumn{2}{|l|}{ NLR } & \multirow[t]{2}{*}{$p$-val } & \multicolumn{2}{|l|}{ PLR } & \multirow[t]{2}{*}{$p$-val } \\
\hline & & & $\begin{array}{l}<4(n=912, \\
74 \%)\end{array}$ & $\begin{array}{l}\geq 4(n=325 \\
26 \%)\end{array}$ & & $\begin{array}{l}<150(n=418 \\
34 \%)\end{array}$ & $\begin{array}{l}\geq 150(n=819, \\
66 \%)\end{array}$ & \\
\hline \multirow[t]{3}{*}{ Province, n (\%) } & Alberta & $595(48)$ & $435(48)$ & $160(49)$ & NS & $226(54)$ & $369(45)$ & $\begin{array}{l}< \\
0.01\end{array}$ \\
\hline & $\begin{array}{l}\text { British } \\
\text { Columbia }\end{array}$ & $255(21)$ & $186(20)$ & $69(21)$ & & $69(17)$ & $186(23)$ & \\
\hline & Ontario & $387(31)$ & $291(32)$ & $96(30)$ & & $123(29)$ & $264(32)$ & \\
\hline \multirow[t]{2}{*}{ Age, years } & $\begin{array}{l}\text { Median } \\
\text { (range) }\end{array}$ & $62(23-88)$ & $61(23-88)$ & $64(24-87)$ & $\begin{array}{l}< \\
0.01\end{array}$ & $62(23-85)$ & $62(23-88)$ & NS \\
\hline & $\geq 65, \mathrm{n}(\%)$ & $482(39)$ & $334(37)$ & $148(46)$ & $\begin{array}{l}< \\
0.01\end{array}$ & $154(37)$ & $328(40)$ & NS \\
\hline Male, n (\%) & & $858(69)$ & $632(69)$ & $226(70)$ & NS & $302(72)$ & $556(68)$ & NS \\
\hline $\mathrm{BMI}, \mathrm{kg} / \mathrm{m}^{2} \mid n=1130$ & $\geq 25, \mathrm{n}(\%)$ & $729(59)$ & $553(61)$ & $176(54)$ & NS & $272(65)$ & $457(56)$ & $\begin{array}{l}< \\
0.01\end{array}$ \\
\hline \multirow[t]{4}{*}{ ECOG PS, n (\%) } & 0 & $524(42)$ & $398(44)$ & $126(39)$ & $\begin{array}{l}< \\
0.01\end{array}$ & $188(45)$ & $336(41)$ & NS \\
\hline & 1 & $466(38)$ & $344(38)$ & $122(38)$ & & $160(38)$ & $306(37)$ & \\
\hline & $2+$ & $62(5)$ & $32(4)$ & $30(9)$ & & $13(3)$ & $49(6)$ & \\
\hline & Unknown & $185(15)$ & $138(15)$ & $47(14)$ & & $57(14)$ & $128(16)$ & \\
\hline \multirow[t]{5}{*}{$\begin{array}{l}\text { Distance from anal verge, } \mathrm{n}(\%) \mid \\
n=1166\end{array}$} & $\begin{array}{l}\text { Median } \\
\text { (range) }\end{array}$ & $6(0-30)$ & $6(0-30)$ & $6(0-25)$ & NS & $6(0-20)$ & $6(0-30)$ & NS \\
\hline & $<5 \mathrm{~cm}$ & $415(34)$ & $306(33)$ & 109 (34) & NS & $137(33)$ & $278(34)$ & NS \\
\hline & $5-10 \mathrm{~cm}$ & $507(41)$ & $381(42)$ & $126(39)$ & & $178(43)$ & $329(40)$ & \\
\hline & $>10 \mathrm{~cm}$ & $244(20)$ & $181(20)$ & $63(19)$ & & $84(20)$ & $160(20)$ & \\
\hline & Unknown & $71(6)$ & $44(5)$ & $27(8)$ & & $19(4)$ & $52(6)$ & \\
\hline \multirow[t]{4}{*}{$\begin{array}{l}\text { Pre-treatment CEA, } \mathrm{n}(\%) \mid n= \\
1093\end{array}$} & $\begin{array}{l}\text { Median } \\
\text { (range) }\end{array}$ & $4(0-1133)$ & $3(0-857)$ & $4(0-1133)$ & NS & $3(0-468)$ & $4(0-1133)$ & NS \\
\hline & $<5 \mathrm{ng} / \mathrm{mL}$ & $667(54)$ & $510(56)$ & $157(48)$ & NS & $230(55)$ & $437(53)$ & NS \\
\hline & $\geq 5 \mathrm{ng} / \mathrm{mL}$ & $426(34)$ & 301 (33) & $125(39)$ & & $142(34)$ & $284(35)$ & \\
\hline & Unknown & $144(12)$ & $101(11)$ & $43(13)$ & & $46(11)$ & $98(12)$ & \\
\hline \multirow[t]{3}{*}{ Clinical stage, n (\%) } & $\|$ & $341(28)$ & $253(28)$ & $88(27)$ & NS & $119(28)$ & $222(27)$ & NS \\
\hline & III & $880(71)$ & $644(71)$ & $236(73)$ & & $291(70)$ & $589(72)$ & \\
\hline & Unknown & $16(1)$ & $15(2)$ & $1(0)$ & & $8(2)$ & $8(1)$ & \\
\hline Hemoglobin (g/dl) & $\begin{array}{l}\text { Median } \\
\text { (range) }\end{array}$ & $\begin{array}{l}136(68- \\
183)\end{array}$ & 137 (68-183) & $131(68-178)$ & $\begin{array}{l}< \\
0.01\end{array}$ & $140(93-183)$ & $133(68-178)$ & $\begin{array}{l}< \\
0.01\end{array}$ \\
\hline
\end{tabular}

BMI Body Mass Index, ECOG PS Eastern Cooperative Oncology Group Performance Status, CEA Carcinoembryonic Antigen, NLR Neutrophil-to-lymphocyte ratio, NS Non-significant, PLR Platelet-to-lymphocyte ratio

index (BMI), statin use, ECOG performance status, pretreatment CEA, clinical stage, distance from anal verge, RT dose ( $<44$ Gy vs $\geq 44$ Gy), type of neoadjuvant chemotherapy (capecitabine vs. 5-fluorouracil vs. other), adjuvant chemotherapy use, baseline hemoglobin, NLR and PLR. Those significant in univariate analysis are listed in Table 3.

On multivariate analyses, independent predictors of shorter DFS were: elevated pre-treatment CEA, clinical stage III/unknown and lower hemoglobin levels, while independent predictors of shorter OS were elevated pretreatment CEA, elevated PS and older age at diagnosis $(\geq$ 65). Independent negative predictors of pCR were elevated pre-treatment CEA and clinical stage III. Elevated baseline NLR and PLR were not independently predictive of pCR, or prognostic for OS or DFS. Multivariate analyses are summarized in Table 4 (DFS), Table 5 (OS) and Table 6 (pCR).

\section{Discussion}

Prognostication and treatment decisions in rectal cancer are primarily based on the anatomic extent of disease spread (i.e. staging), with few biologic tumour or host characteristics (biomarkers) employed to guide decisionmaking in this setting. However, there exists considerable 
Table 2 Treatment details

\begin{tabular}{|c|c|c|c|c|c|c|c|c|}
\hline \multirow[t]{2}{*}{ Variable } & & \multirow{2}{*}{$\begin{array}{l}\text { Total } \\
(N= \\
1237)\end{array}$} & \multicolumn{2}{|l|}{ NLR } & \multirow{2}{*}{$\begin{array}{l}p- \\
\text { val }\end{array}$} & \multicolumn{2}{|l|}{ PLR } & \multirow{2}{*}{$\begin{array}{l}p- \\
\text { val }\end{array}$} \\
\hline & & & $\begin{array}{l}<4(n=912 \\
74 \%)\end{array}$ & $\begin{array}{l}\geq 4(n=325 \\
26 \%)\end{array}$ & & $\begin{array}{l}<150(n=418, \\
34 \%)\end{array}$ & $\begin{array}{l}\geq 150(n=819, \\
66 \%)\end{array}$ & \\
\hline \multirow{4}{*}{$\begin{array}{l}\text { Neoadjuvant chemotherapy, } \mathrm{n} \\
\text { (\%) }\end{array}$} & 5-fluorouracil & $764(62)$ & $558(61)$ & $206(63)$ & \multirow[t]{4}{*}{ NS } & $256(61)$ & $508(62)$ & \multirow[t]{4}{*}{ NS } \\
\hline & Capecitabine & $430(35)$ & $322(35)$ & $108(33)$ & & $149(36)$ & $281(34)$ & \\
\hline & Raltitrexed & $15(1)$ & $14(1)$ & $1(0)$ & & $5(1)$ & $10(1)$ & \\
\hline & Unknown & $28(2)$ & $18(2)$ & $10(3)$ & & $8(2)$ & $20(3)$ & \\
\hline \multirow[t]{5}{*}{ Radiotherapy dose (Gy), n (\%) } & Median (range) & $\begin{array}{l}50(20- \\
80)\end{array}$ & $50(20-74)$ & $50(29-80)$ & NS & $50(20-74)$ & $60(22-80)$ & NS \\
\hline & $<44$ & $36(3)$ & $32(3)$ & $4(1)$ & 0.02 & $15(4)$ & $21(3)$ & \multirow[t]{4}{*}{ NS } \\
\hline & $44-46$ & $225(18)$ & $156(17)$ & $69(21)$ & & $65(15)$ & $160(19)$ & \\
\hline & $\geq 46$ & $966(78)$ & $719(79)$ & $247(76)$ & & $333(80)$ & $633(77)$ & \\
\hline & Unknown & $10(1)$ & $5(1)$ & $5(2)$ & & $5(1)$ & $5(1)$ & \\
\hline \multirow{4}{*}{$\begin{array}{l}\text { Time from nCRT completion to } \\
\text { TME }\end{array}$} & $<6$ weeks & $190(15)$ & $139(15)$ & $51(16)$ & NS & $62(15)$ & $128(16)$ & \multirow[t]{4}{*}{ NS } \\
\hline & $6-12$ weeks & $988(80)$ & $725(80)$ & $263(81)$ & & $335(80)$ & $653(80)$ & \\
\hline & $>12$ weeks & $57(5)$ & $46(5)$ & $11(3)$ & & $20(5)$ & $37(4)$ & \\
\hline & Unknown & $2(0)$ & $2(0)$ & $0(0)$ & & $1(0)$ & $1(0)$ & \\
\hline \multirow[t]{6}{*}{ Adjuvant chemotherapy, n (\%) } & 5-fluorouracil & $214(17)$ & $159(17)$ & $55(17)$ & NS & $78(19)$ & $136(17)$ & \multirow[t]{6}{*}{ NS } \\
\hline & Capecitabine & $336(27)$ & $249(27)$ & $87(27)$ & & $99(24)$ & $237(29)$ & \\
\hline & $\begin{array}{l}\text { 5-fluorouracil / } \\
\text { oxaliplatin }\end{array}$ & $275(22)$ & $199(22)$ & $76(23)$ & & $96(23)$ & $179(22)$ & \\
\hline & $\begin{array}{l}\text { Capecitabine / } \\
\text { oxaliplatin }\end{array}$ & $64(5)$ & $50(5)$ & $14(4)$ & & $32(8)$ & $32(4)$ & \\
\hline & Other & $10(1)$ & $8(1)$ & $2(1)$ & & $4(0)$ & $6(1)$ & \\
\hline & $\begin{array}{l}\text { No adjuvant } \\
\text { chemotherapy }\end{array}$ & $338(27)$ & $247(27)$ & $91(28)$ & & $109(26)$ & $229(28)$ & \\
\hline \multirow[t]{2}{*}{$\begin{array}{l}\text { Completed treatment as } \\
\text { planned, } \mathrm{n}(\%)\end{array}$} & $\begin{array}{l}\text { Neoadjuvant } \\
\text { radiotherapy }\end{array}$ & $\begin{array}{l}1171 \\
(95)\end{array}$ & $859(94)$ & $312(96)$ & NS & $394(94)$ & 777 (95) & NS \\
\hline & $\begin{array}{l}\text { Neoadjuvant } \\
\text { chemotherapy }\end{array}$ & $\begin{array}{l}1001 \\
(81)\end{array}$ & $734(80)$ & $267(82)$ & NS & $333(80)$ & $668(82)$ & NS \\
\hline \multirow[t]{4}{*}{ Type of surgery, n (\%) } & Low anterior resection & $657(53)$ & $490(54)$ & $167(51)$ & NS & $231(55)$ & $426(52)$ & \multirow[t]{4}{*}{ NS } \\
\hline & $\begin{array}{l}\text { Abdominoperineal } \\
\text { resection }\end{array}$ & $535(43)$ & $397(43)$ & $138(42)$ & & $175(42)$ & $360(43)$ & \\
\hline & Pelvic Exenteration & $32(3)$ & $14(2)$ & $18(6)$ & & $9(2)$ & $23(3)$ & \\
\hline & Unknown & $13(1)$ & $11(1)$ & $2(1)$ & & $3(1)$ & $10(1)$ & \\
\hline \multirow[t]{3}{*}{ Total mesorectal excision, n (\%) } & Yes & $\begin{array}{l}1066 \\
(86)\end{array}$ & $783(86)$ & $283(87)$ & NS & $372(89)$ & $694(84)$ & \multirow[t]{3}{*}{ NS } \\
\hline & No & $20(2)$ & $12(1)$ & $8(2)$ & & $7(2)$ & $13(2)$ & \\
\hline & Unknown & $151(12)$ & $117(13)$ & $34(11)$ & & $39(9)$ & $112(14)$ & \\
\hline \multirow[t]{3}{*}{$\begin{array}{l}\text { Circumferential resection } \\
\text { margin, } \mathrm{n}(\%)\end{array}$} & $>1$ mm (uninvolved) & $\begin{array}{l}1061 \\
(86)\end{array}$ & $782(86)$ & $279(86)$ & NS & $358(86)$ & $703(85)$ & \multirow[t]{3}{*}{0.03} \\
\hline & $\leqslant 1 \mathrm{~mm}$ (involved) & $103(8)$ & $72(8)$ & $31(10)$ & & $27(6)$ & $76(9)$ & \\
\hline & Unknown & $73(6)$ & $58(6)$ & $15(5)$ & & $33(8)$ & $40(5)$ & \\
\hline
\end{tabular}

nCRT Neoadjuvant chemoradiotherapy, NLR Neutrophil-to-lymphocyte ratio, NS Non-significant, PLR Platelet-to-lymphocyte ratio, TME Total Mesorectal Excision

heterogeneity in survival and response to treatment even among patients with similar stages of disease, suggesting that differences in host and/or tumour biology may play an important role in determining outcome in these patients. The elucidation of these heterogeneous biological factors may help to guide patient counseling and to personalize management decisions in patients with LARC. The latter may become an increasingly important issue as contemporary, alternative management strategies (including non-operative and 'total' neoadjuvant approaches) 
Table 3 Univariate Analyses

\begin{tabular}{|c|c|c|c|}
\hline Variable & DFS [HR $(95 \% \mathrm{CI})]$ & OS $[\mathrm{HR}(95 \% \mathrm{Cl})]$ & pCR [OR $(95 \% \mathrm{Cl})]$ \\
\hline \multicolumn{4}{|l|}{ NLR } \\
\hline$<4$ & ref & a & a \\
\hline$\geq 4$ & $1.24(1.00-1.53)$ & & \\
\hline \multicolumn{4}{|l|}{ Age at diagnosis } \\
\hline$<65$ & ref & ref & ref \\
\hline$\geq 65$ & $1.34(1.11-1.63)$ & $1.77(1.40-2.22)$ & $0.72(0.53-0.98($ \\
\hline \multicolumn{4}{|l|}{ ECOG PS } \\
\hline 0 & ref & ref & ref \\
\hline 1 & $1.53(1.22-1.93)$ & $1.81(1.36-2.39)$ & $0.72(0.52-1.00)$ \\
\hline $2+$ & $2.06(1.37-3.10)$ & $3.37(2.17-5.25)$ & $0.19(0.06-0.63)$ \\
\hline Unknown & $1.75(1.33-2.31$ & $2.04(1.47-2.83)$ & $0.79(0.51-1.23)$ \\
\hline \multicolumn{4}{|c|}{ Distance from anal verge } \\
\hline$<5 \mathrm{~cm}$ & ref & ref & a \\
\hline $5-10 \mathrm{~cm}$ & $0.92(0.74-1.16)$ & $0.90(0.69-1.18)$ & \\
\hline$>10 \mathrm{~cm}$ & $0.79(0.59-1.05)$ & $0.72(0.51-1.02)$ & \\
\hline Unknown & $1.49(1.02-2.15)$ & $1.52(1.00-2.33)$ & \\
\hline \multicolumn{4}{|c|}{ Pre-treatment CEA (ng/ml) } \\
\hline$<5$ & ref & ref & ref \\
\hline$\geq 5$ & $1.71(1.39-2.11)$ & $1.83(1.43-2.34)$ & $0.37(0.26-0.54)$ \\
\hline Unknown & $1.63(1.21-2.19)$ & $1.49(1.04-2.13)$ & $0.69(0.43-1.12)$ \\
\hline \multicolumn{4}{|l|}{ Clinical stage } \\
\hline$\|$ & ref & ref & ref \\
\hline III & $1.21(0.97-1.52)$ & $1.07(0.82-1.39)$ & $0.66(0.49-0.91)$ \\
\hline Unknown & $3.35(1.75-6.43)$ & $4.14(2.07-8.26)$ & $0.25(0.03-1.92)$ \\
\hline Hemoglobin ${ }^{\mathrm{b}}$ & $0.99(0.99-1.00)$ & $0.99(0.98-1.00)$ & $1.01(1.00-1.02)$ \\
\hline \multicolumn{4}{|c|}{ Neoadjuvant chemotherapy } \\
\hline 5-fluorouracil & ref & ref & a \\
\hline Capecitabine & $1.30(1.06-1.60)$ & $1.27(0.99-1.63)$ & \\
\hline Raltitrexed & $0.56(0.18-1.75)$ & $0.52(0.13-2.08)$ & \\
\hline Unknown & $2.95(1.82-4.76)$ & $3.28(1.90-5.65)$ & \\
\hline \multicolumn{4}{|c|}{ Adjuvant chemotherapy ${ }^{c}$} \\
\hline Not received & ref & ref & N/A \\
\hline Received & $0.63(0.51-0.77)$ & $0.45(0.35-0.56)$ & N/A \\
\hline \multicolumn{4}{|l|}{ Province ${ }^{c}$} \\
\hline Alberta & ref & ref & ref \\
\hline British Columbia & $1.50(1.19-1.90)$ & $1.34(1.02-1.75)$ & $0.56(0.37-0.86)$ \\
\hline Ontario & $0.65(0.51-0.84)$ & $0.47(0.35-0.64)$ & $0.83(0.60-1.16)$ \\
\hline
\end{tabular}

CEA Carcinoembryonic antigen, ECOG PS Eastern Cooperative Oncology Group Performance Status, HR Hazard ratio, OR Odds Ratio Non-significant in univariate analysis: PLR, sex, year of diagnosis, body mass index, statin use, radiation dose

${ }^{\text {a }}$ No statistically significant results

${ }^{\mathrm{b}}$ Measured as continuous variable

${ }^{\mathrm{c}}$ Non-proportional hazards

emerge as potential options for patients with LARC $[1,2$, $12-14]$.

In recent years, the host immune and inflammatory response to malignancy have been demonstrated to be important factors in the development, progression, treatment and survival across a range of cancers [31, 32]. Indeed, tumour-promoting inflammation is a known hallmark of cancer [33]. Accordingly, an increased systemic 
Table 4 DFS Multivariate Analysis

\begin{tabular}{llr}
\hline Outcome & Hazard Ratio $(95 \% \mathrm{Cl})$ & $P$-value \\
\hline NLR & & \\
$<4$ & ref & 0.14 \\
$\geq 4$ & $1.19(0.95-1.50)$ & \\
PLR & & 0.71 \\
$<150$ & ref & \\
$\geq 150$ & $0.96(0.76-1.21)$ & \\
Pre-treatment CEA & & \\
$<5$ ng/ml & ref & \\
$\geq 5$ ng/ml & $1.66(1.34-2.05)$ & 0.01 \\
Unknown & $1.85(1.37-2.51)$ & \\
Clinical stage & & 0.02 \\
II & ref & \\
III & $1.30(1.02-1.65)$ & \\
Unknown & $2.46(1.24-4.90)$ & \\
Hemoglobin & $0.99(0.99-1.00)$ &
\end{tabular}

CEA Carcinoembryonic antigen, DFS Disease-free survival, NLR Neutrophil-tolymphocyte ratio, PLR Platelet-to-lymphocyte ratio

inflammatory response as indicated by a range of surrogate biomarkers (e.g. elevated C-reactive protein, hypoalbuminemia, leukocytosis, thrombocytosis, etc.) - including NLR and PLR - have been shown to be associated with treatment response and outcome in a variety of malignancies,

Table 5 OS Multivariate Analysis

\begin{tabular}{llr}
\hline Outcome & Hazard Ratio $(95 \% \mathrm{Cl})$ & P-value \\
\hline NLR & & \\
$<4$ & ref & \\
$\geq 4$ & $1.00(0.76-1.32)$ & \\
PLR & & 0.59 \\
$<150$ & ref & \\
$\geq 150$ & $0.99(0.76-1.29)$ & \\
Pre-treatment CEA & & $<0.01$ \\
$<5$ ng/ml & ref & \\
$\geq 5$ ng/ml & $1.71(1.33-2.20)$ & \\
Unknown & $1.64(1.13-2.38)$ & \\
ECOG Performance Status & & $<0.01$ \\
0 & ref & \\
1 & $1.43(1.06-1.92)$ & \\
$2+$ & $2.24(1.41-3.56)$ & \\
Unknown & $1.29(0.90-1.85)$ & \\
Age at diagnosis & & \\
$<65$ & ref & \\
$\geq 65$ & $1.50(1.18-1.90)$ & \\
\hline
\end{tabular}

CEA Carcinoembryonic antigen, ECOG Eastern Cooperative Oncology Group, $N L R$ Neutrophil-to-lymphocyte ratio, OS Overall survival, $P L R$ Platelet-to-lymphocyte ratio
Table 6 pCR Multivariate Analysis

\begin{tabular}{llr}
\hline Outcome & Odds Ratio $(95 \% \mathrm{Cl})$ & P-value \\
\hline NLR & & \\
$<4$ & ref & 0.16 \\
$\geq 4$ & $0.76(0.52-1.11)$ & \\
PLR & & 0.90 \\
$<150$ & ref & \\
$\geq 150$ & $1.02(0.73-1.42)$ & \\
Pre-treatment CEA & & \\
$<5$ ng/ml & ref & \\
$\geq 5$ ng/ml & $0.38(0.26-0.55)$ & \\
Unknown & $0.71(0.44-1.14)$ & 0.03 \\
Clinical stage & & \\
II & ref & \\
III & $0.67(0.49-0.92)$ & \\
Unknown & $0.29(0.04-2.29)$ &
\end{tabular}

CEA Carcinoembryonic antigen, ECOG Eastern Cooperative Oncology Group, $N L R$ Neutrophil-to-lymphocyte ratio, $p C R$ Pathologic complete response, $P L R$ Platelet-to-lymphocyte ratio

and several of these have been incorporated into prognostic scoring systems for various types of cancer [34-36]. In addition, some of these factors have also been incorporated into models intended to predict response to treatment [37, 38]. However, the precise mechanisms underlying these observations are complex and remain poorly understood [31].

NLR and PLR are two such biomarkers which are felt to be surrogates of the systemic inflammatory response and are potentially appealing as prognostic and predictive biomarker candidates because they are readily available and easily derived. However, the results of this study suggest that NLR and PLR are neither independently prognostic of outcome nor predictive of response to nCRT in LARC patients undergoing nCRT followed by curative-intent TME.

To our knowledge, this is the largest reported study of the prognostic and predictive impact of NLR and/or PLR in this patient population. Previous studies have reported conflicting results in this setting, with some demonstrating poorer prognosis with higher NLR and/or PLR [39, 40], while others did not observe any significant association [27]. Two prior meta-analyses investigating the prognostic role of NLR and PLR across a range of solid tumours demonstrated an association with adverse OS for both biomarkers in combined study populations of over 40,000 and 12,000 patients, respectively $[19,20]$.

However, these studies included a diverse range of tumour types and included patients with both nonmetastatic and metastatic disease. The results of these meta-analyses may not be generalizable to patients with LARC as a greater association was noted in patients with metastatic disease. Of note, both meta- 
analyses also combined rectal cancer patients with colon cancer patients. There are some data to suggest that the prognostic and predictive capabilities of NLR and PLR differ between rectal and colon cancer as at least one study has demonstrated that NLR was significantly associated with adverse OS in colon, but not rectal, cancer [41]. In addition, it is important to note the potential impact of publication bias favouring positive results in studies of this nature.

With regard to their role in predicting response to neoadjuvant therapy, a recent systematic review and metaanalysis demonstrated an increased likelihood of pCR in patients with rectal cancer and low NLR receiving neoadjuvant chemotherapy +/- radiation (OR 2.01, 95\% CI 1.14-3.55, $p=0.02$ ) [42], which is inconsistent with our results. In addition, a recent study identified elevated PLR (>133.4) to be a significant predictor of poor pathologic response in rectal cancer patients following nCRT [40]. The reasons for these discrepancies are unclear but may be related to differences in study design and analysis. For example, the systematic review included a total of seven studies pertaining to rectal cancer with various inclusion criteria, NLR/PLR cut-offs, and neoadjuvant treatment regimens (including non-radiotherapy-based treatment), while the latter study was not specific to $\mathrm{pCR}$ and did not prespecify cut-off values for the baseline hematologic variables (optimal values were derived from a receiver operating characteristic curve).

Several other prognostic features such as higher stage, poor performance status and elevated pre-treatment CEA emerged as significant prognostic and predictive factors in this cohort, which is consistent with prior studies $[43,44]$.

Strengths of our study include the relatively large sample size, long duration of follow up and multiinstitutional cohort of patients from both academic and community cancer centres across Canada. In addition, the rates of $\mathrm{pCR}$ and 5-year OS and DFS rates observed compare favourably to several landmark trials of nCRT in LARC [3, 4, 45], which further supports the validity and generalizability of our results.

Limitations of our study include the retrospective design, which introduces the potential for unmeasured biases. In addition, approximately one fifth of screened patients were ineligible for inclusion due to missing data, and survival endpoints were not systematically recorded across all provinces, leading to higher proportions of censored in patients in some provinces (e.g. Ontario). Finally, the optimal cut-off values for NLR and PLR are not known and vary widely between studies $[19,20]$. The cutoffs used in this study were chosen based on either the most commonly used or the median cut-off values identified in previously published systematic reviews $[19,20]$. To ascertain that these values were appropriate cut-offs, we also performed cubic spline analyses for both NLR and PLR, which confirmed the suitability of these thresholds (data not shown). In addition, NLR has been shown to have a relatively consistent HR for OS across a range of cut-off values from 1.0-5.0 [20].

\section{Conclusions}

In summary, we did not find any significant prognostic or predictive association for either NLR or PLR in LARC patients undergoing nCRT followed by TME. Ongoing efforts to identify prognostic and/or predictive biomarkers in LARC are warranted and may help to personalize management decisions in this patient population.

\section{Abbreviations}

BMI: Body mass index; CEA: Carcinoembryonic antigen; CHORD: Canadian Health Outcomes Research Database; Cl: Confidence interval; DFS: Diseasefree survival; DR: Distant recurrence; ECOG: Eastern Cooperative Oncology Group; FP: Fluoropyrimidine; LARC: Locally advanced rectal cancer; LR: Local recurrence; NA: Not available; nCRT: Neoadjuvant chemoradiotherapy; NLR: Neutrophil-to-lymphocyte ratio; OS: Overall survival; pCR: Pathological complete response; PLR: Platelet-to-lymphocyte ratio; PS: Performance status; RT: Radiotherapy; TME: Total mesorectal excision

\section{Acknowledgements}

An earlier analysis of this cohort was previously published as an abstract with the following reference:

Dudani S, Marginean H, Tang PA, Monzon JG, Raissouni S, Asmis TR, et al. Neutrophil-to-lymphocyte and platelet-to-lymphocyte ratios as predictive and prognostic markers in patients with locally advanced rectal cancer treated with neoadjuvant chemoradiation. Journal of Clinical Oncology. 2017;35(4_suppl):758.

\section{Authors' contributions}

SD, HM, PT, JM, SR, TA, RG, JG, WC and MV participated in data collection. $\mathrm{SD}, \mathrm{HM}$ and MV analyzed the data and were major contributors in preparing the manuscript. All authors read and approved the final manuscript.

\section{Funding}

This research did not receive any specific grant from funding agencies in the public, commercial, or not-for-profit sectors.

\section{Availability of data and materials}

The datasets used and/or analysed during the current study are available from the corresponding author on reasonable request.

\section{Ethics approval and consent to participate}

This study was approved by the University of British Columbia BC Cancer Research Ethics Board, The University of Ottawa Research Ethics Board and the Alberta Cancer Research Ethics committee (ACREC). For all jurisdictions, informed consent was waived by the respective research ethics boards.

\section{Consent for publication}

Not applicable.

\section{Competing interests}

The authors declare that they have no competing interests.

\section{Author details}

${ }^{1}$ The Ottawa Hospital Cancer Centre/University of Ottawa, Ottawa, Ontario, Canada. ${ }^{2}$ Alberta Health Services/University of Calgary, Calgary, Alberta, Canada. ${ }^{3}$ Alberta Health Services/University of Calgary, Medicine Hat, Alberta, Canada. ${ }^{4}$ British Columbia Cancer Agency, Vancouver, British Columbia, Canada. ${ }^{5}$ Present address: Division of Medical Oncology, Tom Baker Cancer Centre, University of Calgary, 133129 St NW, Calgary, AB T2N 4N2, Canada. 
Received: 9 February 2019 Accepted: 28 June 2019

\section{Published online: 05 July 2019}

\section{References}

1. Glynne-Jones R, Wyrwicz L, Tiret E, et al. Rectal cancer: ESMO clinical practice guidelines for diagnosis, treatment and follow-up. Ann Oncol. 2017;28:iv22-40

2. Benson AB 3rd, Venook AP, Al-Hawary MM, et al. Rectal Cancer, version 2. 2018, NCCN clinical practice guidelines in oncology. J Natl Compr Cancer Netw. 2018;16:874-901

3. Sauer $\mathrm{R}$, Becker $\mathrm{H}$, Hohenberger W, et al. Preoperative versus postoperative chemoradiotherapy for rectal cancer. N Engl J Med. 2004;351:1731-40.

4. O'Connell MJ, Colangelo LH, Beart RW, et al. Capecitabine and oxaliplatin in the preoperative multimodality treatment of rectal cancer: surgical end points from National Surgical Adjuvant Breast and bowel project trial R-04. J Clin Oncol. 2014;32:1927-34.

5. Park IJ, You YN, Agarwal A, et al. Neoadjuvant treatment response as an early response indicator for patients with rectal cancer. J Clin Oncol. 2012;30:1770-6.

6. Fokas $E$, Liersch $T$, Fietkau $R$, et al. Tumor regression grading after preoperative chemoradiotherapy for locally advanced rectal carcinoma revisited: updated results of the CAO/ARO/AIO-94 trial. J Clin Oncol. 2014;32:1554-62.

7. Patel UB, Taylor F, Blomqvist $L$, et al. Magnetic resonance imaging-detected tumor response for locally advanced rectal cancer predicts survival outcomes: MERCURY experience. J Clin Oncol. 2011;29:3753-60.

8. Garcia-Aguilar J, Chen Z, Smith DD, et al. Identification of a biomarker profile associated with resistance to neoadjuvant chemoradiation therapy in rectal cancer. Ann Surg. 2011;254:486-92; discussion 492-3.

9. Capirci C, Valentini V, Cionini L, et al. Prognostic value of pathologic complete response after neoadjuvant therapy in locally advanced rectal cancer: long-term analysis of 566 ypCR patients. Int J Radiat Oncol Biol Phys. 2008;72:99-107.

10. Habr-Gama A, Perez RO, Nadalin W, et al. Operative versus nonoperative treatment for stage 0 distal rectal cancer following chemoradiation therapy: long-term results. Ann Surg. 2004;240:711-7; discussion 717-8.

11. Maas M, Beets-Tan RG, Lambregts DM, et al. Wait-and-see policy for clinical complete responders after chemoradiation for rectal cancer. J Clin Oncol. 2011;29:4633-40.

12. Appelt AL, Ploen J, Harling $H$, et al. High-dose chemoradiotherapy and watchful waiting for distal rectal cancer: a prospective observational study. Lancet Oncol. 2015;16:919-27.

13. Renehan AG, Malcomson L, Emsley R, et al. Watch-and-wait approach versus surgical resection after chemoradiotherapy for patients with rectal cancer (the OnCoRe project): a propensity-score matched cohort analysis. Lancet Oncol. 2016:17:174-83.

14. Cercek A, Roxburgh CSD, Strombom P, et al. Adoption of Total neoadjuvant therapy for locally advanced rectal Cancer. JAMA Oncol. 2018:4:e180071.

15. Chan J, Kinsella MT, Willis JE, et al. A predictive genetic signature for response to Fluoropyrimidine-based neoadjuvant Chemoradiation in clinical stage II and III rectal Cancer. Front Oncol. 2013;3:288.

16. Kuremsky JG, Tepper JE, McLeod HL. Biomarkers for response to neoadjuvant chemoradiation for rectal cancer. Int J Radiat Oncol Biol Phys. 2009;74:673-88.

17. Dayde D, Tanaka I, Jain R, et al. Predictive and prognostic molecular biomarkers for response to neoadjuvant Chemoradiation in rectal Cancer. Int J Mol Sci. 2017;18:573

18. Armstrong D, Raissouni S, Price Hiller J, et al. Predictors of pathologic complete response after neoadjuvant treatment for rectal Cancer: a multicenter study. Clin Colorectal Cancer. 2015;14:291-5.

19. Templeton AJ, Ace O, McNamara MG, et al. Prognostic role of platelet to lymphocyte ratio in solid tumors: a systematic review and meta-analysis. Cancer Epidemiol Biomark Prev. 2014;23:1204-12.

20. Templeton AJ, McNamara MG, Seruga B, et al. Prognostic role of neutrophilto-lymphocyte ratio in solid tumors: a systematic review and meta-analysis. J Natl Cancer Inst. 2014;106:dju124.

21. Shin US, You YN, Price BA, et al. Is the neutrophil-lymphocyte ratio (NLR) a predictive and prognostic factor in rectal cancer patients treated with neoadjuvant chemoradiation (nCRT)? J Clin Oncol. 2016:34:3605

22. Carruthers $R$, Tho LM, Brown J, et al. Systemic inflammatory response is a predictor of outcome in patients undergoing preoperative chemoradiation for locally advanced rectal cancer. Color Dis. 2012;14:e701-7.
23. Krauthamer M, Rouvinov K, Ariad S, et al. A study of inflammation-based predictors of tumor response to neoadjuvant chemoradiotherapy for locally advanced rectal cancer. Oncology. 2013;85:27-32.

24. Kim IY, You SH, Kim YW. Neutrophil-lymphocyte ratio predicts pathologic tumor response and survival after preoperative chemoradiation for rectal cancer. BMC Surg. 2014;14:94

25. Picardo SL, Teo M, Jalil KIA, et al. Correlation between platelet/lymphocyte ratio, neutrophil/lymphocyte ratio and response to neoadjuvant chemoradiation therapy in rectal cancer. J Clin Oncol. 2016;34:e15140.

26. Toiyama $Y$, Inoue $Y$, Kawamura M, et al. Elevated platelet count as predictor of recurrence in rectal cancer patients undergoing preoperative chemoradiotherapy followed by surgery. Int Surg. 2015;100:199-207.

27. Portale G, Cavallin F, Valdegamberi A, et al. Platelet-to-lymphocyte ratio and neutrophil-to-lymphocyte ratio are not prognostic biomarkers in rectal Cancer patients with curative resection. J Gastrointest Surg. 2018;22:1611-8.

28. Lee $\mathrm{H}$, Hwang S, Lee SJ, et al. Systemic inflammatory response after preoperative Chemoradiotherapy can affect oncologic outcomes in locally advanced rectal Cancer. Anticancer Res. 2017;37:1459-65.

29. Edge SB, Compton CC. The American joint committee on Cancer: the 7th edition of the AJCC cancer staging manual and the future of TNM. Ann Surg Oncol. 2010;17:1471-4.

30. Durrleman S, Simon R. Flexible regression models with cubic splines. Stat Med. 1989;8:551-61.

31. Grivennikov SI, Greten FR, Karin M. Immunity, inflammation, and cancer. Cell. 2010;140:883-99.

32. Coussens LM, Werb Z. Inflammation and cancer. Nature. 2002:420:860-7.

33. Hanahan D, Weinberg RA. Hallmarks of cancer: the next generation. Cell. 2011;144:646-74.

34. McMillan DC. The systemic inflammation-based Glasgow prognostic score: a decade of experience in patients with cancer. Cancer Treat Rev. 2013;39:534-40.

35. Li Y, Jia H, Yu W, et al. Nomograms for predicting prognostic value of inflammatory biomarkers in colorectal cancer patients after radical resection. Int J Cancer. 2016:139:220-31.

36. Heng DY, Xie W, Regan MM, et al. Prognostic factors for overall survival in patients with metastatic renal cell carcinoma treated with vascular endothelial growth factor-targeted agents: results from a large, multicenter study. J Clin Oncol. 2009:27:5794-9.

37. Mezquita L, Auclin E, Ferrara R, et al. Association of the Lung Immune Prognostic Index with Immune Checkpoint Inhibitor Outcomes in patients with advanced non-small cell lung Cancer. JAMA Oncol. 2018;4:351-7.

38. Ferrucci PF, Gandini S, Battaglia A, et al. Baseline neutrophil-to-lymphocyte ratio is associated with outcome of ipilimumab-treated metastatic melanoma patients. Br J Cancer. 2015;112:1904-10.

39. Shen $L$, Zhang $H$, Liang $L$, et al. Baseline neutrophil-lymphocyte ratio ( $>/=2$. 8) as a prognostic factor for patients with locally advanced rectal cancer undergoing neoadjuvant chemoradiation. Radiat Oncol. 2014;9:295.

40. Kim TG, Park W, Kim H, et al. Baseline Neutrophil-Lymphocyte Ratio and Platelet-Lymphocyte Ratio in Rectal Cancer Patients Following Neoadjuvant Chemoradiotherapy. Tumori J. 2018. https://doi.org/10.1177/ 0300891618792476

41. Rashtak S, Ruan X, Druliner BR, et al. Peripheral neutrophil to lymphocyte ratio improves prognostication in Colon Cancer. Clin Colorectal Cancer. 2017;16:115-123 e3.

42. Li X, Dai D, Chen B, et al. The value of neutrophil-to-lymphocyte ratio for response and prognostic effect of neoadjuvant chemotherapy in solid tumors: a systematic review and meta-analysis. J Cancer. 2018;9:861-71.

43. Park JW, Lim S-B, Kim DY, et al. Carcinoembryonic antigen as a predictor of pathologic response and a prognostic factor in locally advanced rectal Cancer patients treated with preoperative Chemoradiotherapy and surgery. Int J Radiat Oncol Biol Phys. 2009;74:810-7.

44. Das $\mathrm{P}, \mathrm{Crane} \mathrm{CH}$. Staging, prognostic factors, and therapy of localized rectal cancer. Curr Oncol Rep. 2009;11:167-74.

45. Allegra CJ, Yothers G, O'Connell MJ, et al. Neoadjuvant 5-FU or Capecitabine plus radiation with or without Oxaliplatin in rectal Cancer patients: a phase III randomized clinical trial. J Natl Cancer Inst. 2015;107(11):djv248. https:// doi.org/10.1093/jnci/djv248

\section{Publisher's Note}

Springer Nature remains neutral with regard to jurisdictional claims in published maps and institutional affiliations. 